\title{
EVIDÊNCIAS DA EFICIÊNCIA DE GASTOS PÚBLICOS NA ALOCAÇÃO DOS RECURSOS DESTINADOS AO ENSINO FUNDAMENTAL NOS ESTADOS BRASILEIROS
}

\author{
EVIDENCES OF THE EFFICIENCY OF PUBLIC \\ EXPENDITUREIN THE ALLOCATION OF RESOURCES FOR \\ FUNDAMENTAL EDUCATION IN THE BRAZILIAN STATES
}

\section{EVIDENCIAS DE LA EFICIENCIA DEL GASTO PUBLICO EM LA ALOCAÇÃO DE LOS RECURSOS DESTINADOS A LA ENSEÑO BÁSICA EM LOS ESTADOS BRASILEÑOS}

\author{
Herivélton Antônio Schuster \\ Mestre em Contabilidade pela Universidade Regional de \\ Blumenau (FURB), Brasil / Docente de Ciências \\ Contábeis da Faculdade Mater Dei, Brasil \\ herivelton_schuster@hotmail.com \\ Vinicius Zonatto \\ Professor permanente do Programa de Pós-Graduação \\ em Ciências Contábeis da Universidade Regional de \\ Blumenau (FURB), Brasil / Pós-doutor em Ciências \\ Contábeis pela Universidade do Vale do Rio dos Sinos \\ (UNISINOS), Brasil \\ viniciuszonatto@gmail.com
}

\author{
Contextus \\ ISSNe 2178-9258 \\ Organização: Comitê Científico Interinstitucional \\ Editor Científico: Carlos Adriano Santos Gomes \\ Avaliação: double blind review pelo SEER/OJS \\ Edição de texto e de layout: Carlos Daniel Andrade \\ Recebido em 13/12/2016 \\ Aceito em 12/09/2017 \\ $2^{a}$ versão aceita em 19/10/2017
}

\section{RESUMO}

Este artigo tem como objetivo avaliar a eficiência dos gastos públicos com a educação nas séries iniciais do ensino fundamental, tomando como universo de análise o conjunto de 260 municípios brasileiros, composto pelos dez maiores municípios de cada Estado, levando em consideração os mais populosos segundo a base de dados do Instituto Brasileiro de Geografia e Estatística (IBGE). A análise foi efetuada por meio da aplicação da técnica Análise Envoltória de Dados - DEA, sendo a metodologia mais adequada para medir a eficiência da alocação dos recursos públicos diante dos diversos serviços prestados. Os resultados evidenciaram 13 municípios com eficiência máxima na alocação dos recursos, sendo que 92,31\% destes também atingiram a meta do Índice de Desenvolvimento da educação Básica -IDEB em sua totalidade. Ficou evidente ainda que os municípios com maior custo por aluno matriculado e localizados em regiões com maior desenvolvimento econômico mostraramse menos eficientes.

Palavras-chave: Educação. Gastos Públicos. IDEB. DEA.

\begin{abstract}
The objective this article is to evaluate the efficiency of public spending on education in the initial grades of education elementary, taking as a universe of analysis the set of 260 Brazilian municipalities, composed of the ten largest municipalities of each State, taking into account the most populous according to Database of the Brazilian Institute of Geography and Statistics (IBGE). The analysis was performed through the application of the Data Envelopment Analysis (DEA) technique, and the most adequate methodology is to measure the efficiency of the allocation of public resources to the various services provided. The results showed 13 municipalities with maximum efficiency in the allocation of resources, and $92.31 \%$ of these also reached the goal of the Development Index of Basic Education - IDEB in its entirety. It became evident that the municipalities with the highest cost per student enrolled and located in regions with greater economic development were less efficient.
\end{abstract}

Keywords: Education. Public spending. IDEB. DEA. 


\section{RESUMEN}

Este artículo tiene como objetivo evaluar la eficiencia del gasto público en educación en los primeros grados de la escuela primaria, tomando como universo de los 260 municipios, que consiste en los diez municipios de cada estado, teniendo en cuenta la más poblada de acuerdo con la la base de datos del Instituto brasileño de Geografía y Estadística (IBGE). El análisis se realizó mediante la aplicación de análisis envolvente de datos técnicos DEA, la metodología más adecuada para medir la eficiencia de la asignación de los recursos públicos en los diferentes servicios prestados. Los resultados mostraron 13 municipios con la máxima eficiencia en la asignación de recursos, y 92,31\% de ellos también llegaron a la meta Índice de Desarrollo de la Educación Básica - IDEB en su totalidad. Se hizo evidente que las ciudades con el mayor costo por estudiante inscrito y se encuentra en las regiones con mayor desarrollo económico eran menos eficientes.

Palabras clave: Educación. El gasto público. IDEB. DEA.

\section{INTRODUÇÃO}

As disparidades socioeconômicas regionais no Brasil impõem desafios à União quanto ao repasse de receitas tributárias para os demais entes federados (Estados e Municípios), destinado a reduzir eventuais desequilíbrios entre a capacidade de arrecadação local e a demanda por bens e serviços públicos. Nesse sentido, a Constituição Federal de 1988 ampliou a participação dos níveis estadual e municipal no uso de verbas federais, principalmente, por meio do Fundo de Participação dos estados (FPE) e dos Municípios (FPM) (SOUZA JUNIOR; GASPARINI, 2006).

No que tange à gestão da educação, as políticas públicas lhe têm dedicado atenção especial, levando em consideração sua relevância para desenvolver o ensino no país (PORTELA, 2006). Uma educação de qualidade reflete-se na construção das características pessoais dos indivíduos, no aprimoramento de suas várias habilidades, bem como em sua contribuição para a formação da sociedade (SILVA, 2013). Em suma, consiste no alicerce para o desenvolvimento econômico e social de um país. Tendo em vista sua importância, o governo brasileiro tem investido de forma contínua no setor, com políticas que visam aumentar o nível de escolaridade da população e melhorar as condições para isso (SAVIAN; BEZERRA, 2013).

Ao governo são impostas três funções econômicas básicas: alocar recursos; ajustar e distribuir renda; e estabilizar a economia. A primeira se norteia pela ideia de que o fornecimento de bens públicos não pode se restringir ao mercado, mas cabe também ao estado. A função distributiva, por sua vez, parte de uma estipulação da renda adequada à sociedade em questão. Por último, a função estabilizadora objetiva o controle da produção, do emprego, dos preços e do equilíbrio no balanço de pagamentos, além do alcance de taxas apropriadas de crescimento econômico (RIANI, 2002). 
Conforme destacam Savian e Bezerra (2013), um dos principais objetivos da economia é alocar os recursos, mesmo que limitados, no atendimento de necessidades ilimitadas. $\mathrm{O}$ governo os prospecta com base nos impostos e então analisa a forma mais eficiente para a alocação. A eficiência desse processo, principalmente na educação, desafia e instiga os responsáveis a buscar alternativas que promovam a maximização de resultados sociais. Nesse contexto, destaca-se a função do governo em oferecer bens e serviços públicos e semipúblicos, que não cabem à iniciativa privada, devido à sua inviabilidade econômica (SILVA et al. 2012). Rezende, Slomski e Corrar (2005) afirmam que a alocação de recursos em educação, saúde e habitação objetivam a redução das desigualdades sociais e a melhoria de qualidade de vida da população.

O desafio mencionado levou o Brasil a adotar, em 2005, o Índice de Desenvolvimento da Educação Básica (IDEB), com a intenção de avaliar a evolução da aprendizagem no país. Segundo Fernandes (2005), o IDEB evidencia o fluxo escolar e as médias de desempenho dos anos iniciais e finais do ensino fundamental. Com isso, monitora a qualidade do sistema educacional ao combinar fluxo e aprendizagem escolar.
Tal combinação pode variar de 0 a 10, em âmbito nacional, federal e municipal. Os resultados do IDEB de 2013 mostraram grande discrepância nos resultados das escolas entre si e dos estados entre si. Em evidência, os estados das regiões norte e nordeste alcançaram os índices mais baixos em relação às outras regiões do país (MINISTÉRIO DA EDUCAÇÃO, 2013).

Uma questão que emerge desse contexto refere-se à análise da eficiência e da eficácia dos gastos públicos em educação no país. Apesar da importância do tema, poucos estudos têm se dedicado a avaliar os aspectos de eficiência dos gastos públicos em âmbito nacional. Desse modo, considerando a oportunidade de estudo identificada na literatura, estabeleceu-se a seguinte questão de pesquisa: qual a eficiência na alocação dos recursos destinados ao ensino fundamental nos maiores municípios dos estados brasileiros? Com o intuito de responder à questão apresentada, o objetivo deste estudo consiste em analisar a eficiência na alocação dos recursos destinados ao ensino fundamental nos dez maiores municípios de cada estado.

A análise da eficiência foi elaborada com o uso da Análise Envoltória dos Dados (DEA). De acordo com Savian e Bezerra (2013), essa metodologia permite construir fronteiras de eficiência 
para detectar situações de ineficiência. Assim, sugere-se a possibilidade de melhorar o desempenho dos municípios ineficientes, sem a necessidade de melhorar a quantidade de recursos alocados ao setor, mas apenas alocando-os de forma mais eficiente. Segundo Coelli et al. (2005), o método oferece medida da eficiência relativa entre as entidades estudadas.

O estudo justifica-se pela representatividade do ensino fundamental no país, bem como pela oportunidade de avaliar a eficiência dos gastos públicos realizados nessa subárea da educação. Também fornece evidências que permitem identificar municípios mais eficientes como benchmarks, os quais podem estimular a realização de novos estudos, com o propósito de investigar práticas de gestão adotadas que melhor contribuem para o alcance de resultados próximos. Conforme Andere e Araújo (2008), o estudo da eficiência dos recursos públicos aplicados na educação permite analisar sua destinação de modo a promover justiça social. A alocação adequada possibilita o acesso à educação e, como consequência, a redução de desigualdades sociais, bem como a promoção do desenvolvimento socioeconômico.

Diante do exposto, considerando a importância dos serviços públicos essenciais para a promoção de uma melhor qualidade de vida da população, como a educação, torna-se apropriada a realização desta pesquisa, com a intenção de analisar a eficiência quanto à alocação de recursos públicos na educação fundamental, de forma a disponibilizar aos municípios pesquisados dados a auxiliar no estabelecimento de novas práticas de políticas públicas.

\section{FUNDAMENTAÇÃO TEÓRICA}

Este capítulo consiste em apresentar a revisão literária direcionada ao estudo: inicialmente, sobre aspectos da educação e, posteriormente, sobre a Análise Envoltória dos Dados (DEA). Para tal fundamentação, utilizou-se de pesquisa realizada nas bases Scopus, Portal Capes, Google Acadêmico, Spell, Scielo e ScienceDirect. Para a busca dos estudos relacionados, utilizaram-se as palavraschave "gastos públicos", "DEA", “alocação de recursos públicos”, "gastos com ensino fundamental" e "educação básica”. Os resultados são apresentados na sequência.

\subsection{Aspectos da Educação}

A educação, conforme dispõe a Constituição Federal de 1988 (CF), caracteriza-se como direito social que o 
poder público tem a obrigação de prover gratuitamente à população. Conforme disposto no artigo 208, a educação básica é um direito obrigatório para crianças e adolescentes, devendo ser oferecida de forma gratuita, sob pena de responsabilização do governante que não o fizer (BRASIL, 1988).

A CF estabelece ainda, em seu artigo 212, a obrigatoriedade dos municípios, dos estados e da União de alocarem um mínimo de recursos captados por meio de impostos (receitas) na área da educação, sendo o mínimo de 18\% para a União e $25 \%$ para os estados e municípios (BRASIL, 1988). Aos municípios cabe prioritariamente a obrigação do ensino fundamental e da educação infantil.

Para Zoghbi et al. (2009), a educação ganhou extrema importância para o desenvolvimento do ser humano, da sociedade e da economia de uma nação. Além disso, a educação pública possui a função de redistribuição de riqueza, ampliando as condições de mobilidade social (WILBERT; D'ABREU, 2013). Wilbert e D'Abreu (2013) caracterizam a educação como um bem público não puro, que pode ser objetivo de política pública a fim de melhorar a eficiência quanto à alocação dos recursos, dando ênfase à maior oferta de educação e por consequência originando um maior equilíbrio nas desigualdades. Conforme ressaltam Silva, Souza e Araújo (2013), a educação de qualidade torna-se elemento essencial para melhores condições de vida do indivíduo e para o desenvolvimento do ambiente em que está inserido.

A Lei de Diretrizes e Bases da Educação Nacional (BRASIL, 1996), no artigo $3^{\circ}$, antevê os princípios que norteiam a educação brasileira, sendo um deles a garantia do padrão de qualidade (inciso IX). Ora, a qualidade provém de um processo de construção lenta, que exige mudança na forma de pensar e de agir dos indivíduos, sendo resultado da evolução histórica em que o conceito assumiu diversos significados, diferentes para cada geração ou época analisada. Sobre essa tensão entre a garantia legal do padrão de qualidade e a complexa construção efetiva deste, lançam luz as palavras de Portela (2006).

Para ela, as necessidades dos alunos estão geralmente centradas na convivência social e na aprendizagem da leitura e dos cálculos, sendo um dos maiores desafios para o processo de ensino. No entanto, existe certo descompasso entre, de um lado, a legislação e a política de financiamento e, do outro, o padrão de qualidade, a prática docente e as evidentes necessidades dos alunos do ensino fundamental (PORTELA, 2006). A 
propósito, Schmelkes (1994) pontua que a qualidade almejada para a educação básica requer uma série de competências necessárias, como o domínio de aspectos culturais, a capacidade de resolução de problemas e a continuidade da aprendizagem, além da formação de valores e atitudes que estejam de acordo com as novas exigências sociais. Conforme Paro (1997), as dificuldades ao implementar uma escola de qualidade devem-se ao fato de ela estar pautada em padrões tradicionais, característica essa da escola pública, que atendia às camadas privilegiadas de décadas passadas. Tal descompasso pode impactar negativamente na qualidade do ensino prestado.

Segundo Arelaro et al. (2004), as discussões referentes à forma como a educação é financiada no Brasil devem levar em conta a situação socioeconômica e algumas especificidades na forma de arrecadar e aplicar impostos no país. Os recursos, nesse sentido, podem ser afetados pelo desempenho das políticas econômica, tributária e fiscal, que determinam tanto a arrecadação quanto a aplicação de recursos financeiros na educação local (ARELARO et al., 2004).

No que se refere ao Índice de Desenvolvimento da Educação Básica (IDEB), Fernandes (2007) explica que tal indicador educacional visa monitorar a qualidade dos sistemas por meio da combinação entre fluxo e aprendizagem escolar. Na mensuração do fluxo escolar, o IDEB apresenta médias de desempenho que variam de 0 a 10, dos anos iniciais e finais do ensino fundamental, permitindo inferir sobre o avanço ou não dos sistemas educacionais, em âmbito nacional, federal e municipal (FERNANDES, 2007). Nesse contexto, a busca pela eficiência requer aspectos como melhorar a relação resultados/custos, ou seja, maximizar os resultados (outputs) e minimizar os custos (inputs) (WILBERT; D’ABREU, 2013).

Para Diniz e Corrar (2011), o equilíbrio entre arrecadação e alocação dos recursos é um dos principais desafios para os entes federativos. A melhoria da eficiência no setor público exige, primordialmente, definir de quem é responsabilidade pela arrecadação de cada tipo de tributo e de provisão de cada serviço público, a fim de buscar o equilíbrio almejado. Brunet, Bertê e Borges (2008) inferem que a manutenção ou a obtenção de eficiência decorrem do equilíbrio entre o gasto e os critérios de qualidade, sem esquecer que melhoras na educação requerem investimentos de longo prazo. Com isso, os gastos com educação devem proporcionar a eficiência e a igualdade nos sistemas de ensino, proporcionando assim um melhor 
desempenho e o desenvolvimento dos alunos.

Nesta pesquisa, a quantificação e avaliação da eficiência da alocação dos recursos em educação fundamental nos casos analisadas baseia-se na metodologia Data Envelopment Analysis (DEA).

\subsection{Análise Envoltória dos Dados (DEA)}

Segundo Lins e Meza (2000), a aplicação da metodologia DEA compõe-se de três etapas principais: i) a definição das DMUs (decision making units) para análise (em nosso caso, municípios); ii) a definição das variáveis de insumo (inputs) e de produto (outputs), relevantes para estabelecer a eficiência das DMUs; e iii) a aplicação dos modelos DEA, com maior ou menor nível de sofisticação. Para Wilbert e D’Abreu (2013), uma das vantagens do método é a possibilidade de utilizar múltiplos inputs e outputs sem que a análise se torne complexa.

Essa metodologia mede a eficiência de unidades produtivas que atuam em uma mesma área levando em consideração a distância entre os DMUs de sua respectiva fronteira de eficiência, determinados a partir dos dados da produção do conjunto de unidades (SILVA, 2008; FONSECA; FERREIRA, 2009). Baseia-se em uma amostra de dados analisados a diferentes DMUs, cujo objetivo é classificar as unidades produtoras em eficientes ou ineficientes (MARINHO, 2003).

Assim, conforme Mello et. al. (2005), vale ressaltar que eficiência é a relação entre o que foi produzido, levando em consideração os recursos disponíveis pelas DMUs, e o que poderia ter sido produzido, utilizando os mesmos recursos. Difere de eficácia, que na visão dos autores, está atrelada apenas ao que foi produzido, não levando em consideração o volume de recursos utilizados e consumidos para tal.

A eficiência estimada pela metodologia DEA é relativa, apesar de baseada em contextos reais. Silva et al. (2012) frisam que, por meio de um modelo empírico, o DEA identifica escores de eficiência para cada unidade de produção, de forma a avaliar uma unidade em relação às outras de um conjunto homogêneo. Dessa forma, as análises originam uma fronteira de eficiência, em que cada unidade tomadora de decisão (DMU) avaliada terá um escore de eficiência entre 0 e 1 (entre $0 \%$ e $100 \%$ ). No entanto, uma restrição imposta ao método é que a DMU deve estar sobre a fronteira de eficiência ou dentro dela (FARIA; JANNUZZI; SILVA, 2008; SILVA et al., 2012) 
O modelo DEA é composto por três subgrupos: i) CCR, modelo clássico com retornos constantes à escala, onde as DMUs são comparadas entre si independentemente da estrutura física em que cada uma funciona; ii) $\mathrm{BCC}$, modelo clássico com retornos variáveis à escala, em que as unidades ineficientes são definidas por meio da comparação entre as DMUs, considerando o mesmo tamanho de operação; iii) modelo aditivo e multiplicativo (HAAS, 2003; SILVA et. al., 2009).

Quanto aos aspectos do BCC utilizado neste estudo, a DMU eficiente será a que contiver o menor valor a determinado input ou o maior valor a determinado output. Contudo, no método DEA, qualquer modelo de DMU determina seu próprio conjunto de pesos, com o intuito de transparecer o melhor possível em relação aos outros, possibilitando a cada DMU um conjunto de pesos diferente (MELLO et. al., 2005).

Silva et al. (2012) ressaltam que a DEA torna-se a metodologia mais adequada para medir a eficiência da alocação dos recursos públicos diante dos diversos serviços prestados, pois identifica o desempenho das unidades de análise e, ao mesmo tempo, compara-as, possibilitando identificar as melhores práticas de políticas públicas, bem como possíveis melhorias quanto à qualidade de bens e serviços prestados.

Conforme Marinho (2003), o desempenho dos DMUs, neste caso os municípios, são medidos por meio da comparação de seus resultados, confrontando as quantidades geradas de seus diferentes produtos, a partir dos recursos utilizados, com os resultados e os consumos das outras DMUs da amostra, sendo ineficiente então a DMU que gerar menor quantidade de produtos do que outra com o mesmo consumo.

Neste estudo, a aplicação da metodologia DEA possibilita evidenciar os municípios mais e menos eficientes no que tange à alocação dos recursos na educação, permitindo, portanto, a identificação daqueles benchmarks. A seguir são revisitados alguns estudos anteriores desenvolvidos sob esta temática.

\subsection{Estudos relacionados}

Júnior e Gasparini (2006) avaliaram em que medida o Fundo de Participação dos estados (FPE) atende aos objetivos redistributivos traçados pela Constituição Federal, bem como o seu impacto sobre a eficiência da gestão pública. Utilizaram na aferição a Análise de Componentes Principais (ACP) e a DEA e consideraram as 27 unidades federativas. Inicialmente, os 
autores construíram um limite de melhor disponibilidade de serviços para avaliar a equidade distributiva entre os estados. A seguir, utilizaram uma função custo para obter um índice de eficiência dos gastos públicos. Por fim, estimaram uma fronteira que descreve a eficiência arrecadatória. Nos resultados encontrados, a região Sul apresentou a melhor disponibilidade de serviços e a maior eficiência no uso dos recursos. Em contraste, o Nordeste apresentou fortes carências e também grande desperdício. Os achados permitiram aos autores concluir que todos os estados necessitam de complementação de verbas, constatando distorções presentes no atual critério observado.

Faria, Jannuzzi e Silva (2008) apresentaram uma revisão de várias aplicações de DEA nas políticas públicas do estado do Rio de Janeiro. Aplicaram o modelo BCC da DEA, visando maximizar os outputs sem redução dos inputs. Utilizaram como inputs indicadores de despesas per capita com educação e cultura, bem como com saúde e saneamento. Em contrapartida, as variáveis outputs foram: a taxa de alfabetização de 10 a 14 anos, a proporção de domicílios particulares permanentes com esgotamento sanitário adequado, a proporção de domicílios particulares permanentes com saneamento adequado, o inverso da taxa de mortalidade por causas hídricas e a proporção de crianças de dois a cinco anos matriculadas em creches ou escolas de educação infantil. Como resultado, pode-se destacar, por boas práticas na alocação de recursos ou pelas condições de renda média, os municípios de São Gonçalo, Japeri, Queimados, Cantagalo, São João de Meriti e Resende.

Zoghbi et al. (2009) avaliaram o desempenho e a eficiência relativa das 27 unidades federativas, no que diz respeito aos gastos que fazem em educação nos níveis fundamental e médio. Inicialmente, os autores construíram alguns indicadores de desempenho com base em variáveis quantitativas e qualitativas. Os resultados obtidos indicam que estados com melhor desempenho não necessariamente são os mais eficientes. Além disso, para a grande maioria dos estados pesquisados, existe um amplo espaço para melhorar a eficiência gestora.

Savian e Bezerra (2013) avaliaram a eficiência dos gastos públicos municipais do Paraná com as séries iniciais do ensino fundamental, no período de 2005 e 2009. Para tanto, os municípios foram classificados quanto à eficiência dos gastos no setor, propiciando a comparação também entre as mesorregiões do estado. Os resultados mostraram que, na maioria dos municípios paranaenses, os gastos 
públicos com educação são ineficientes, o que demonstra a necessidade de revisão, por parte da administração pública, dos meios de alocação dos recursos.

$$
\text { Wilbert e D'Abreu }
$$
avaliaram a eficiência dos gastos públicos com educação fundamental dos municípios alagoanos, por meio da DEA, no período de 2007 a 2011. Como variáveis utilizaram o Produto Interno Bruto (PIB), o número de habitantes, o número de estudantes matriculados, o gasto com educação e as notas do IDEB. Os municípios classificados como eficientes foram aqueles com as piores condições de partida, em termos de riqueza média e nível educacional, e que gastaram pouco por aluno matriculado. Por sua vez, os municípios menos eficientes foram aqueles com a melhor condição de partida em termos de PIB per capita e que apresentaram elevados gastos por aluno, mas que alcançaram os piores desempenhos no IDEB de 2011.

O Quadro 1 apresenta uma síntese dos principais resultados dos estudos correlatos identificados na revisão da literatura. 
Quadro 1 - Principais resultados dos estudos relacionados

\begin{tabular}{|c|l|c|}
\hline Autores & \multicolumn{1}{|c|}{ Principais resultados } & $\begin{array}{c}\text { Nível } \\
\text { geográfico }\end{array}$ \\
\hline $\begin{array}{c}\text { Júnior e } \\
\text { Gasparini } \\
(2006)\end{array}$ & $\begin{array}{l}\text { A região Sul apresentou a maior eficiência no uso dos recursos. Todos os } \\
\text { estados necessitam de complementação de verbas. }\end{array}$ & $\begin{array}{c}27 \text { estados } \\
\text { brasileiros. }\end{array}$ \\
\hline $\begin{array}{c}\text { Faria, Jannuzzi } \\
\text { e Silva (2008) }\end{array}$ & $\begin{array}{l}\text { A eficiência das políticas públicas está relacionada a ocorrência de boas } \\
\text { práticas na alocação dos recursos e condições de renda média. }\end{array}$ & $\begin{array}{c}\text { Estado do Rio } \\
\text { de Janeiro }\end{array}$ \\
\hline $\begin{array}{c}\text { Zoghbi } \text { et al. } \\
\text { (2009) }\end{array}$ & $\begin{array}{l}\text { Estados com melhor desempenho não necessariamente são os mais } \\
\text { eficientes. A maior parte dos estados pode melhorar a eficiência da gestão } \\
\text { dos recursos. }\end{array}$ & $\begin{array}{c}27 \text { estados } \\
\text { brasileiros. }\end{array}$ \\
\hline $\begin{array}{c}\text { Savian e } \\
\text { Bezerra (2013) }\end{array}$ & $\begin{array}{l}\text { Na maioria dos municípios paranaenses pesquisados os gastos públicos com } \\
\text { educação são ineficientes. }\end{array}$ & $\begin{array}{c}\text { Estado do } \\
\text { Paraná }\end{array}$ \\
\hline $\begin{array}{c}\text { Wilbert e } \\
\text { D'Abreu } \\
\text { (2013) }\end{array}$ & $\begin{array}{l}\text { Municípios eficientes foram aqueles com as piores condições de partida, em } \\
\text { termos de riqueza média e nível educacional, e que gastaram pouco por } \\
\text { melhor condição de partida em termos de PIB per capita e que apresentaram } \\
\text { elevados gastos por aluno. }\end{array}$ & $\begin{array}{c}\text { Estado de } \\
\text { Alagoas }\end{array}$ \\
\hline
\end{tabular}

Fonte: dados da pesquisa.

Frente aos resultados expostos no Quadro 1, percebe-se a predominância de pesquisas realizadas enfatizando os investimentos realizados em nível estadual (JÚNIOR; GASPARINI, 2006; FARIA; JANNUZZI; SILVA, 2008; ZOGHBI et al., 2009). Apenas dois estudos, Savian e Bezerra (2013) e Wilbert e D'Abreu (2013), investigaram a eficiência dos gastos públicos com a educação nas séries iniciais do ensino fundamental com abrangência em âmbito municipal, observando municípios dos estados do Paraná e Alagoas, respectivamente. Ademais, fora a revelação de ineficiência da maioria dos municípios pesquisados, os resultados encontrados apresentam resultados conflitantes, portanto inconclusivos. Assim, torna-se oportuna a investigação proposta nesta pesquisa.

\section{PROCEDIMENTOS METODOLÓGICOS}

A pesquisa caracteriza-se como descritiva, realizada por meio de análise documental e abordagem quantitativa dos dados. Para Andrade (2004), uma pesquisa descritiva busca observar fatos, registrálos, analisá-los, classificá-los e interpretálos, sem que o pesquisador efetue interferências. Quanto a uma pesquisa quantitativa, seu principal objetivo é, segundo Martins e Theóphilo (2007), o de avaliar a população ou os fenômenos estudados por meio de técnicas estatísticas.

A população do estudo compreende 260 municípios brasileiros, caracterizados como os 10 maiores em população de cada unidade da federação, segundo a base de dados do Instituto Brasileiro de Geografia e Estatística (IBGE). Ressalta-se que 2 
municípios foram excluídos da amostra, por não possuírem todos os dados necessários para compor o banco de dados utilizados nesta pesquisa. Dessa forma, a amostra ficou composta por 258 municípios, representando 99,23\% da população inicial. Visto que o total equivale a 85.561.841 de habitantes e a população do Brasil, em 2013, totalizou 201.032.714 (IBGE), a razão entre a população da amostra e a do país foi de $0,426 \%$.

Quanto às variáveis utilizadas nesta investigação, foram divididas em inputs (insumos) e outputs (produtos). Para avaliar a eficiência dos gastos públicos no ensino fundamental, foram utilizados os seguintes inputs (insumos): i) DLiq despesas liquidadas alocadas ao Ensino Fundamental da Educação Básica; ii) GMed - gasto médio por aluno matriculado; iii) DEdu - índice IDHM medido pela escolaridade da população adulta e pelo fluxo escolar da população jovem; iv) DRen - índice IDHM medido pela renda mensal per capita. Como output (produto), utilizou-se o IMed - Média do IDEB. O Quadro 2 relaciona os inputs e outputs utilizados no estudo.

Quadro 2 - Variáveis utilizadas

\begin{tabular}{|c|c|c|}
\hline Insumos/Inputs & Descrição dos inputs & Base de dados \\
\hline $\begin{array}{l}\text { DLiq - Despesas } \\
\text { Liquidadas }\end{array}$ & $\begin{array}{l}\text { Recursos públicos alocados ao ensino } \\
\text { fundamental da educação básica. }\end{array}$ & $\begin{array}{c}\text { Sítio do Tesouro Nacional } \\
\text { Brasileiro (STN) }\end{array}$ \\
\hline GMed - Gasto Médio & Gasto médio por aluno matriculado. & $\begin{array}{c}\text { Resultado da divisão: Despesas } \\
\text { Liquidadas / Alunos Matriculados }\end{array}$ \\
\hline $\begin{array}{c}\text { DEdu - IDHM - } \\
\text { Dimensão Educação }\end{array}$ & $\begin{array}{l}\text { O Índice de Desenvolvimento Humano dos } \\
\text { Municípios (IDHM), dimensão educação, é } \\
\text { medido pela escolaridade da população adulta e } \\
\text { pelo fluxo escolar da população jovem. O } \\
\text { índice varia de } 0 \text { a 1: quanto maior, melhor. }\end{array}$ & $\begin{array}{l}\text { Sítio do Programa das Nações } \\
\text { Unidas para o Desenvolvimento } \\
\text { (PNUD) }\end{array}$ \\
\hline $\begin{array}{l}\text { DRen - IDHM - } \\
\text { Dimensão Renda }\end{array}$ & $\begin{array}{l}\text { No IDHM, a dimensão renda é medida pela } \\
\text { renda mensal per capita. O índice varia de } 0 \text { a } \\
\text { 1: quanto maior, melhor. }\end{array}$ & Sítio do PNUD \\
\hline Produto/Output & Descrição do output & Base de dados \\
\hline $\begin{array}{l}\text { IMed - Nota média } \\
\text { do IDEB }\end{array}$ & Média do IDEB (Séries iniciais $-1^{\mathrm{a}}$ a $4^{\mathrm{a}}$ ). & $\begin{array}{c}\text { Sítio do Instituto Nacional de } \\
\text { Estudos e Pesquisas Educacionais } \\
\text { Anísio Teixeira (INEP) }\end{array}$ \\
\hline
\end{tabular}

Fonte: dados da pesquisa.

Os gastos públicos totais com educação de cada município em 2013 e as despesas liquidadas foram extraídos de relatório divulgado pelo sítio do Tesouro Nacional (MINISTÉRIO DA FAZENDA, 2015). O número total de matrículas no ensino fundamental foi obtido do Instituto Nacional de Estudos e Pesquisas Educacionais Anísio Teixeira (INEP, 2015), compondo o índice os alunos matriculados em escola municipal no ano de 2013. Quanto às variáveis DEdu e 
DRen, foram coletadas a partir do Ranking do IDH 2010 dos estados, através do Programa das Nações Unidas para o Desenvolvimento (PNUD, 2015).

As notas no IDEB das escolas municipais (outputs) são de 2013, resultantes da avaliação aplicada aos alunos da $4^{\mathrm{a}}$ e $8^{\mathrm{a}}$ série do ensino fundamental, foram divulgadas pelo Ministério da Educação (MINISTÉRIO DA EDUCAÇÃO, 2015). Não se utilizaram as notas de $5^{\mathrm{a}}$ a $8^{\mathrm{a}}$ séries porque, dentre as relativas a todos os municípios, somente estavam disponíveis as da rede estadual pública.

A análise dos dados foi realizada por meio da DEA, com aplicação do modelo BCC orientado ao output. As análises da eficiência na alocação dos recursos da educação no ensino fundamental foram realizadas por meio do software MaxDEA 5®. Os resultados da pesquisa são apresentados a seguir.

\section{ANÁLISE DOS DADOS}

O estudo foi efetuado nos 10 maiores municípios de cada estado do Brasil. Entretanto, durante a coleta de dados, verificou-se que os municípios de Alagoinhas (BA) e Cantá (RR) não continham dados suficientes para compor a variáveis Nota Média do IDEB (IMed), sendo então excluídos da amostra. As notas do IDEB de 2013 dos anos iniciais do ensino fundamental $\left(1^{\circ}\right.$ ao $5^{\circ}$ ano, que compreende a classe de alfabetização até a $4^{\mathrm{a}}$ Série) foram divulgadas em Setembro de 2014 pelo Ministério da Educação (MEC). Também, como regularmente se procede, a projeção de metas para o IDEB é divulgada, o que auxilia no comparativo das notas obtidas com as projetadas para cada município.

Referente ao alcance da meta do IDEB, conforme dados disponíveis no sítio do INEP, observou-se que apenas 84 municípios $(32,56 \%)$ da amostra analisada não alcançaram as metas propostas para as séries iniciais. Destes, 1 município $(1,19 \%)$ está localizado no Acre, 6 em Alagoas (7,14\%), 6 no Amapá (7,14\%), 5 na Bahia (5,95\%), 2 no Ceará (2,38\%), 3 no Espirito Santo (3,57\%), 1 em Goiás (1,19\%), 7 no Maranhão (8,33\%), 3 no Mato Grosso $(3,57 \%), 2$ no Mato Grosso do Sul (2,38\%), 4 em Minas Gerais (4,76\%), 3 no Pará (3,57\%), 1 na Paraíba (1,19\%), 7 em Pernambuco (8,33\%), 3 no Piauí $(3,57 \%)$, 7 no Rio de Janeiro (8,33\%), 3 no Rio Grande do Norte (3,57\%), 5 no Rio Grande do Sul (5,95\%), 1 em Rondônia (1,19\%), 4 em Roraima (4,76\%), 4 em São Paulo $(4,76 \%), 5$ em Sergipe $(5,95 \%)$ e 1 no Tocantins (1,19\%). Esses resultados estão apresentados no Quadro 3. 
Quadro 3 - Municípios que não alcançaram a meta do IDEB 2013

\begin{tabular}{|c|c|c|c|c|c|}
\hline \multicolumn{6}{|c|}{ Estado / Município } \\
\hline $\mathrm{AC}$ & Cruzeiro do Sul & MA & Codó & RJ & Niterói \\
\hline $\mathrm{AL}$ & Maceió & MA & Açailândia & RJ & Belford Roxo \\
\hline $\mathrm{AL}$ & Palmeira dos Índios & MA & Balsas & RJ & Campos dos Goytacazes \\
\hline $\mathrm{AL}$ & Rio Largo & MT & Várzea Grande & RJ & São João de Meriti \\
\hline $\mathrm{AL}$ & União dos Palmares & MT & Rondonópolis & RJ & Petrópolis \\
\hline $\mathrm{AL}$ & São Miguel dos Campos & MT & Cáceres & $\mathrm{RN}$ & Natal \\
\hline $\mathrm{AL}$ & Delmiro Gouveia & MS & Corumbá & RN & Parnamirim \\
\hline AP & Macapá & MS & Sidrolândia & $\mathrm{RN}$ & São Gonçalo do Amarante \\
\hline AP & Oiapoque & MG & Contagem & $\mathrm{RS}$ & Porto Alegre \\
\hline AP & Mazagão & MG & Juiz de Fora & $\mathrm{RS}$ & Pelotas \\
\hline AP & Porto Grande & MG & Betim & $\mathrm{RS}$ & Gravataí \\
\hline AP & Tartarugalzinho & MG & Ribeirão das Neves & $\mathrm{RS}$ & Viamão \\
\hline AP & Calçoene & PA & Bragança & $\mathrm{RS}$ & São Leopoldo \\
\hline $\mathrm{BA}$ & Feira de Santana & PA & Marituba & RO & Porto Velho \\
\hline $\mathrm{BA}$ & Vitória da Conquista & PA & Barcarena & RR & Caracaraí \\
\hline BA & Lauro de Freitas & PB & Santa Rita & RR & Bonfim \\
\hline BA & Jequié & $\mathrm{PE}$ & Recife & RR & Amajari \\
\hline BA & Teixeira de Freitas & $\mathrm{PE}$ & Olinda & RR & Normandia \\
\hline $\mathrm{CE}$ & Juazeiro do Norte & $\mathrm{PE}$ & Caruaru & SP & São Paulo \\
\hline $\mathrm{CE}$ & Maranguape & $\mathrm{PE}$ & Paulista & SP & Santo André \\
\hline ES & Vila Velha & $\mathrm{PE}$ & Cabo de Santo Agostinho & SP & Osasco \\
\hline $\mathrm{ES}$ & Serra & $\mathrm{PE}$ & Camaragibe & SP & Mauá \\
\hline ES & Vitória & $\mathrm{PE}$ & Vitória de Santo Antão & SE & Nossa Senhora do Socorro \\
\hline GO & Formosa & PI & Teresina & SE & Itabaiana \\
\hline MA & São Luís & PI & Piripiri & SE & São Cristóvão \\
\hline MA & Imperatriz & PI & União & SE & Estância \\
\hline MA & São José de Ribamar & RJ & São Gonçalo & SE & Tobias Barreto \\
\hline MA & Timon & RJ & Nova Iguaçu & TO & Tocantinópolis \\
\hline
\end{tabular}

Fonte: dados da pesquisa.

Todos os demais municípios selecionados para compor a amostra (174) atingiram ou superaram as metas estabelecidas para 2013. No Amazonas (AM), Paraná (PR) e Santa Catarina (SC), todos os municípios pesquisados contemplaram tal resultado.

Considerando a aplicação dos recursos realizados pelas capitais, comparativamente ao valor total investido nos estados correspondentes, verificou-se que os recursos alocados especificamente para educação do ensino fundamental representam:
- em Rio Branco

$(\mathrm{AC})$

( $\mathrm{R} \$ 98.562 .356,31), 41,29 \%$ do total;

- em Maceió (AL) (R\$ 163.722.899,92), $33,31 \%$;

- em Macapá (AP) (R \$ 130.596.832,50), $56,20 \%$;

- em Manaus (AM) (R\$684.312.525,94), $66,18 \%$;

- em Salvador (BA) $(\mathrm{R} \$ 225.932 .524,54)$, $22,51 \%$

- em Fortaleza (CE) (R\$ 612.660.058,08), $47,38 \%$;

- em Vitória (ES) (R\$ 168.052.039,58), $21,50 \%$ 
- em Goiânia (GO) (R\$ 588.721.159,58), $45,59 \%$;

- em

São

Luís

(MA)

( $\mathrm{R} \$ 334.325 .254,88), 37,39 \%$;

- em Cuiabá (MT) (R\$ 250.804.631,30), $45,86 \%$;

- em Campo Grande $(\mathrm{R} \$ 337.205 .717,38), 47,49 \%$;

- em Belo Horizonte ( $\mathrm{R} \$ 818.825 .489,37), 44,78 \%$;

- em Belém do Pará (R\$ 295.830.208,62), 26,22\%;

- em João Pessoa ( $\mathrm{R} \$ 299.707 .062,72), 49,28 \%$;

- em Curitiba (PR) (R\$ 651.298.208,75), $45,06 \%$;

- em Recife (PE) (R \$ 557.954.788,71), $49,13 \%$;

- em Teresina (PI) (R\$ 211.805.128,54), $47,56 \%$;

- no Rio de Janeiro ( $\mathrm{R} \$ 2.927 .059 .130,98), 64,50 \%$;

- em Natal (RN) (R\$211.303.646,43), $39,86 \%$;

- em Porto Alegre

(RS)

( $\mathrm{R} \$ 329.995 .854,00), 26,80 \%$;

- em Porto Velho (RO) ( R\$ 169.512.937,06), 48,20\%;

- em Boa Vista (RR) (R\$ 136.761.489,66), 71,89\%;

- em Florianópolis

$(\mathrm{R} \$ 173.756 .837,70), 20,53 \%$;
- em São Paulo

(SP)

( R \$ 2.829.508.389,07), 63,77\%;

- em Aracaju (SE) (R\$ 133.978.576,05), $34,30 \%$;

- e, em Palmas

$(\mathrm{R} \$ 111.243 .187,28), 38,61 \%$.

Esses resultados revelam uma variação significativa na participação dos investimentos entre as capitais dos estados brasileiros. Do mesmo modo, permitem avaliar a representatividade dos recursos em comparação com os demais municípios considerados maiores em população: há uma disparidade de investimentos em educação fundamental não apenas entre as regiões, mas entre, de um lado, as capitais e, de outro, os municípios mais populosos.

Vale ressaltar que os recursos alocados são relativos e dependentes da quantidade de alunos matriculados no ensino fundamental em cada município. Apesar de tais variações percentuais, os achados identificados nesta pesquisa evidenciam não haver grandes discrepâncias entre os municípios pesquisados quanto ao gasto médio por aluno. Nesse contexto, mesmo com significativo percentual de recursos alocados, alguns dos municípios não alcançaram a meta proposta pelo IDEB, como é o caso de Maceió (AL), Macapá (AP), Vitória (ES), São Luís (MA), Recife (PE), Teresina (PI), Natal (RN), Porto 
Alegre (RS), Porto Velho (RO) e São Paulo (SP). Tais resultados revelam ineficiência na alocação de tais gastos públicos.

De maneira geral, verificou-se que $73,08 \%$ dos municípios pesquisados que tiveram os maiores custos por aluno matriculado, sendo um para cada Estado, atingiram a meta do IDEB. Eis a relação detalhada:

- Rio Branco (AC): gasto médio de $\mathrm{R} \$ 10.384,82$;

- Pedra Branca do Amapari (AP): gasto médio de R\$ 8.040,68;

- Tefé (AM): gasto médio de $\mathrm{R} \$ 5.185,41$;

- Itabuna (BA): gasto médio de $\mathrm{R} \$ 5.576,00$;

- Fortaleza (CE): gasto médio de $\mathrm{R} \$ 4.532,55$;

- Goiânia (GO): gasto médio de $\mathrm{R} \$ 8.358,12$;

- Cuiabá (MT): gasto médio de $\mathrm{R} \$ 8.889,68$;

- Paranaíba (MS): gasto médio de $\mathrm{R} \$ 7.682,47$;

- Belo Horizonte (MG): gasto médio de $\mathrm{R} \$$ 6.820,82;

- Belém do Pará (PA): gasto médio de $\mathrm{R} \$$ 6.886,66;

- João Pessoa (PB): gasto médio de $\mathrm{R} \$ 7.822,80$;
- Foz do Iguaçu (PR): gasto médio de $\mathrm{R} \$ 7.334,92$;

- Floriano (PI): gasto médio de $\mathrm{R} \$ 4.875,76$;

- Duque de Caxias (RJ): gasto médio de $\mathrm{R} \$ 7.809,46$;

- Ji-Paraná (RO): gasto médio de $\mathrm{R} \$ 6.889,95$

- Pacaraima (RR): gasto médio de $\mathrm{R} \$ 6.523,17$;

- Lages (SC): gasto médio de $\mathrm{R} \$ 8.008,44$;

- São José dos Campos (SP): gasto médio de $\mathrm{R} \$ 8.421,49$ e;

- Aracaju (SE): gasto médio de $\mathrm{R} \$ 7.654,61$.

Constatou-se também que atingiram a média 76,92\% dos municípios, sendo um para cada Estado, com menor custo por aluno matriculado.

Neste contexto, faz-se necessário avaliar a eficiência dos municípios quanto à alocação dos recursos públicos com a finalidade de ofertar um ensino fundamental de qualidade e atingir os objetivos propostos. Assim, foram calculados os escores para cada município por meio do método DEA. A Tabela 1 contempla a análise descritiva dos escores de eficiência apurados nesta investigação. 
Tabela 1 - Análise descritiva dos escores de eficiência

\begin{tabular}{ccccccc}
\hline Variável & Mínimo & Máximo & Média & Desvio-Padrão & Assimetria & Curtose \\
\hline Escore de Eficiência & 0,470 & 1,000 & 0,7397 & 0,12316 & 0,312 & $-0,362$ \\
\hline
\end{tabular}

Fonte: dados da pesquisa.

Verifica-se uma média dos escores de eficiência na ordem de $73,97 \%$, assim como moderada dispersão entre o máximo e o mínimo, além de alto desvio padrão entre os escores dos municípios analisados. Tais aspectos evidenciam as disparidades existentes quanto à gestão dos recursos públicos destinados à educação. Torna-se relevante analisar o coeficiente assimetria positivo (0,312), que demonstra a existência de escores de eficiência acima da média, e de curtose negativo, confirmando a dispersão existente acerca da média.

Foram aplicados testes de correlação:

- entre a variável DLiq e o total de alunos matriculados, obteve-se coeficiente positivo em 0,976 ;
- $\quad$ entre o DLiq e DEdu, obteve-se 0,275; e

- $\quad$ entre o DLiq e DRen, 0,340.

Ambos os testes foram significativos a um nível de $5 \%$, visto que foram de 0,00 , resultado este que condiz com as condições de significância para uma correlação estatística. De acordo com Hair Jr. et al. (2005), o mínimo para uma interpretação aceitável é 0,05, tornando-se aceito para tais correlações estatísticas.

Os dados referentes à análise da eficiência dos municípios da amostra são apresentados a seguir, estando descritos na Tabela 2 os que obtiveram a eficiência máxima (1).

Tabela 2 - Eficiência máxima dos municípios pesquisados

\begin{tabular}{|c|c|c|c|c|c|}
\hline छ & Município & 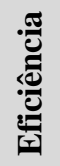 & है & Município & 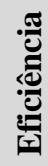 \\
\hline 1 & Alta Floresta (MT) & 1 & 8 & Jaboatão dos Guararapes (PE) & 1 \\
\hline 2 & Alto Alegre (RR) & 1 & 9 & Ji-Paraná (RO) & 1 \\
\hline 3 & Amajari (RR) & 1 & 10 & Maringá (PR) & 1 \\
\hline 4 & Brasiléia (AC) & 1 & 11 & Ouro Preto do Oeste (RO) & 1 \\
\hline 5 & Cacoal (RO) & 1 & 12 & Pimenta Bueno (RO) & 1 \\
\hline 6 & Feijó (AC) & 1 & 13 & Sobral (CE) & 1 \\
\hline 7 & Guaraí (TO) & 1 & & & \\
\hline
\end{tabular}

Fonte: dados da pesquisa. 
Dos 258 municípios analisados, apenas 13 alcançaram eficiência máxima. Com base no trabalho de Savian e Bezerra (2013), optou-se por estruturar a disposição dos demais municípios analisados em categorias, com base nos graus de ineficiência: baixa, moderada e forte. Nesse contexto, foram classificadas como eficientes as DMUs com $\theta=1$. DMUs em que $0,8 \leq \theta<1$ foram classificadas com ineficiência fraca; com ineficiência moderada, aquelas com $0,6 \leq \theta$ $<0,8$; e com ineficiência forte, no caso de $\theta<0,6$. Uma síntese dos resultados pode ser evidenciada na Tabela 3.

Tabela 3 - Níveis de Eficiência e Percentuais

\begin{tabular}{lcc}
\hline Níveis de Eficiência & Municípios \\
\hline Eficientes $(\theta=1)$ & 13 & $5,04 \%$ \\
\hline Ineficiência Fraca $(0,8 \leq \theta<1)$ & 57 & $22,09 \%$ \\
\hline Ineficiência Moderada $(0,6 \leq \theta<0,8)$ & 154 & $59,69 \%$ \\
\hline Ineficiência Forte $(0<\theta<0,6)$ & 34 & $13,18 \%$ \\
\hline Total & $\mathbf{2 5 8}$ & $\mathbf{1 0 0 \%}$ \\
\hline
\end{tabular}

Fonte: dados da pesquisa.

A partir da Tabela 3, é possível visualizar os municípios que alcançaram a eficiência máxima, isto é, localizaram-se na fronteira de eficiência projetada. Com $100 \%$, foram identificados 13 municípios (Alta Floresta, Alto Alegre, Amajari, Brasiléia, Cacoal, Feijó, Guaraí, Jaboatão dos Guararapes, Ji-Paraná, Maringá, Ouro Preto do Oeste, Pimenta Bueno, Sobral), correspondendo a $5,04 \%$ da amostra. Destes, apenas Amajari (RR) não atingiu a meta do IDEB 2013. Quanto a sua distribuição, 4 estão localizados em Rondônia (RO), 2 em Roraima (RR), 2 no Acre (AC), 1 em Mato Grosso (MT), 1 no Paraná (PR), 1 em Tocantins (TO), 1 em Pernambuco (PE) e 1 no Ceará (CE).

Esses resultados divergem dos achados encontrados no estudo desenvolvido por Júnior e Gasparini (2006), em que a região Sul apresentou a melhor disponibilidade de serviços e a maior eficiência no uso dos recursos. No entanto, corrobora os resultados encontrados no estudo desenvolvido por Savian e Bezerra (2013), evidenciando que, na maioria dos municípios paranaenses, os gastos públicos com educação são ineficientes. Considerando os resultados da amostra analisada de maneira consolidada, pode-se verificar que $72,87 \%$ dos municípios apresentaram ineficiência moderada ou forte, resultados que convergem com os achados de Zoghbi et al. (2009), que constataram que, na maior parte dos estados brasileiros, é possível melhorar a eficiência da gestão dos recursos públicos investidos em educação. 
Os 13 municípios que apresentaram maior eficiência são benchmarks para o desenvolvimento dos demais. Como exemplo extremo, a cidade de Feira de Santana (BA), que foi a mais ineficiente, deve orientar-se pelas 13 cidades eficientes. No entanto, todos os municípios ineficientes devem basear-se naqueles que lhes servem de benchmark, no sentido de conhecer suas potencialidades e planejar suas estratégias de ação para alcançarem maiores níveis de eficiência.

Quanto aos municípios com potencial para atingir eficiência, ou seja, aqueles com ineficiência fraca, identificaram-se 57, correspondendo a $22,09 \%$ do total. Apresentaram ineficiência moderada 154 municípios, que representam $59,69 \%$ do total dos casos analisados, e $13,18 \%$ do total correspondem aos 34 municípios ineficientes fortes. Contudo, 46,90\% da amostra (121 municípios) apresentaram desempenho superior à média $(73,97 \%$ de eficiência), e o desempenho mínimo da amostra foi baixo, sendo de 47,39\% de eficiência. Esses resultados convergem com os de Zoghbi et al. (2009), pois sinalizam que os estados com melhor desempenho econômico não necessariamente atingem a melhor eficiência. Também revelam que grande parte dos estados possuem lacunas de melhorias quanto à eficiência, necessitando de melhores práticas de gestão dos recursos.

Para o alcance das metas propostas pelo IDEB 2013, os 19 municípios com nível de eficiência entre $90 \%$ e $99,99 \%$, portanto mais próximos da fronteira de eficiência, deveriam ter obtido pontuações maiores: respectivamente, de 0,13 pontos para Sena Madureira (AC), Campo Alegre (AL) 0,154 pontos, Tarauacá (AC) 0,14 pontos, Sinop (MT) 0,198 pontos, Nova Andradina (MS) 0,25 pontos, Senador Guiomard (AC) 0,226 pontos, Naviraí (MS) 0,273 pontos, Foz do Iguaçu (PR) 0,344 pontos, Tangará da Serra (MT) 0,279 pontos, Joinville (SC) 0,38 pontos, Colatina (ES) 0,32 pontos, Paraíso do Tocantins (TO) 0,372 pontos, Plácido de Castro (AC) 0,342 pontos, Barra do Garças (MT) 0,414 pontos, Rio Verde (GO) 0,577 pontos, Currais Novos (RN) 0,454 pontos, Iguatu (CE) 0,5 pontos, Guajará-Mirim (RO) 0,504 pontos e Sousa (PB) 0,55 pontos.

Tal resultado evidencia um desfavorável contexto, pois $94,96 \%$ dos municípios pesquisados apresentaram certo grau de ineficiência, menor ou maior, podendo ser contestado que o princípio da eficiência não tem sido devidamente imposto ou cumprido em relação à alocação de recursos para a educação. 
Assim, torna-se necessário que esses municípios revejam a metodologia adotada para a destinação dos recursos. Conforme Almeida (2001), o maior desafio aos entes federativos é o de encontrar mecanismos que, mesmo com a capacidade limitada de expansão do gasto com educação, possam garantir melhoria na qualidade de ensino/aprendizado por meio do gasto por aluno na educação básica.

\section{CONSIDERAÇÕES FINAIS}

Com o objetivo de analisar a eficiência dos dez maiores municípios de cada estado brasileiro na alocação de recursos públicos para o provimento do serviço de educação básica, utilizou-se a DEA. A amostra pesquisada possui características distintas, tais como atividade econômica, localização geográfica, cultura regional, clima, dentre outros aspectos capazes de influenciar no resultado se comparados individualmente. Contudo, foram analisados os dados de 258 municípios, de todas as mesorregiões brasileiras, com o intuito de classificá-los pelo nível de eficiência e indicar aqueles que são benchmarks, no que se refere à alocação de recursos públicos para o provimento do serviço de educação básica.

Os resultados revelaram um nível de eficiência relativamente baixo dos governos quanto à gestão dos recursos. Apenas 13 municípios (5,04\% da amostra) alcançaram eficiência máxima de 100\%. Os demais $(72,87 \%$ da amostra) apresentaram um moderado e forte grau de ineficiência. No entanto, 5,03\% (19 municípios) beiravam a fronteira de eficiência. Nesse sentido, verificou-se a existência de peculiaridades no serviço disponibilizado para uma significante parcela da população, fato que corroborou os achados de Wilbert e D'Abreu (2013), em que os municípios mais eficientes foram os que menos gastaram por aluno matriculado. Em contrapartida, os municípios com maior custo por aluno matriculado se apresentaram entre os menos eficientes.

Verificou-se que os municípios mais eficientes são relativamente pequenos, se comparados aos demais que compunham a amostra de seu respectivo estado, alguns apresentando ainda baixo nível de atividade econômica, porém atingindo o objetivo de ofertar acesso à educação com eficiência para toda ou a maioria de sua população, o que se reflete positivamente em indicadores sociais como o IDHM nas dimensões de educação e renda. Conforme Faria, Jannuzzi e Silva (2008), pode-se atingir tal resultado com a eficiência das políticas públicas, a boa alocação dos recursos públicos destinados 
à educação ou as condições de renda média.

Em contrapartida, muitos municípios identificados como menos eficientes são grandes em população, sendo alguns destes o maior do seu estado, com elevado nível de atividade econômica e com igual ou maior porcentagem de recursos alocados na educação. Porém, não eficientes ao ofertar o acesso à educação a suas respectivas populações, demonstrando que a aplicação de grande quantidade de recursos não necessariamente implica eficiência na oferta desse serviço. Com efeito, uma parcela dos municípios investigados $(26,92 \%)$ que gastaram mais recursos não atingiram eficiência no fornecimento dos serviços educacionais. Nesse contexto, constantemente se discute, em projetos de lei que direcionam maior aporte de recursos financeiros para a educação, qual a melhor forma de alocálos.

Em contrapartida, dentre os municípios com menos recursos, apenas $23,08 \%$ não atingiram eficiência. Tal fato pode ser explicado nos termos de Delgado e Machado (2008): apesar das disparidades socioeconômicas existentes no país, aspectos como maior destinação de assessoria técnica e financeira aos municípios colaboram para a melhor alocação de recursos, cabendo para os municípios mais desenvolvidos políticas de ajuste e readequação. Por sua vez, para os menos desenvolvidos, o acréscimo marginal nos insumos poderá contribuir para a obtenção de ganhos proporcionais e o alcance de melhor eficiência.

É importante ponderar sobre a defasagem do efeito dos gastos públicos sobre os indicadores de educação. Este estudo analisou a eficiência com base em gastos de 2013. Todavia, os impactos da educação são cumulativos. Assim, investimentos realizados naquele período poderão resultar em melhoria nos índices avaliados em períodos futuros. Investimentos relacionados a capacitação e treinamento de professores, a desenvolvimento de materiais didáticos adequados ou a adoção de novas tecnologias no ambiente de ensino podem ser mais demorados, considerando a realidade distinta dos municípios brasileiros. Portanto, podem levar anos para poder abranger a maior parte dos professores da primeira e da segunda séries do ensino fundamental, o que também tende a refletir-se na qualidade dos gastos já realizados e nos indicadores de eficiência futuros. Adicionalmente, como os professores são servidores públicos, o conhecimento tende a permanecer na rede de educação. Desse modo, um bom rendimento escolar nas séries iniciais do 
ensino fundamental deverá repercutir em melhores resultados nas séries subsequentes.

Mesmo apresentando algumas limitações que impedem a generalização de tais resultados, as evidências encontradas nesta pesquisa ampliam a oportunidade de avaliar a eficiência dos municípios brasileiros na alocação de recursos públicos, com vistas ao provimento do serviço de educação básica de qualidade.
Os achados propiciam uma avaliação sob uma perspectiva nacional, revelando resultados encontrados em realidades distintas e por vezes conflitantes. Também emergem aqui oportunidades para a realização de outros estudos, por exemplo, aplicando o mesmo ou outro escopo de pesquisa em um espaço temporal maior e analisando a evolução dos gastos municipais. 


\section{REFERÊNCIAS}

ALMEIDA, I. C. Gastos com educação no período de 1994 a 1999. Revista brasileira de estudos pedagógicos, v. 82, n. 200/201, p. 202, 2001.

ANDERE, M. A.; ARAÚJO, A. M. P. de. Aspectos da formação do professor de ensino superior de Ciências Contábeis: uma análise dos programas de pós-graduação. Revista de Contabilidade e Finanças, v. 19, n. 48, p. 91-102, 2008.

ANDRADE, M. M. de. Como preparar trabalhos para cursos de pós-graduação: noções práticas. 6. ed. São Paulo: Atlas, 2004.

ARELARO, L. R. G.; HORODYNSKI-MATSUSHIGUE, L. B.; HELENE, O.; CAMARGO, R. B. Passando a limpo o financiamento da educação nacional: algumas considerações. Revista da USP, p. 30-42, 2004.

ATLAS do Desenvolvimento Humano no Brasil, IDH-M e ICV: Metodologia. O Programa das Nações Unidas para o Desenvolvimento (PNUD). Disponível em: <www.pnud.org.br/IDH/Default.aspx?indiceAccordion=1\&li=li_AtlasMunicipios $>$. Acesso em: 14 jul. 2015.

BRASIL. Constituição da República Federativa do Brasil: 1988. Disponível em: <www.planalto.gov.br>. Acesso em: 13 jul. 2015.

BRASIL. Lei de Diretrizes e Bases da Educação (LDB), Lei ${ }^{\circ}$ 9.394, de 20 dezembro de 1996.

BRUNET, J. F. G.; BERTÊ, A. M. de A.; BORGES, C. B. Qualidade do gasto público em educação nas redes públicas estaduais e municipais. In: $2^{\circ}$ Congresso Consad de Gestão Pública. Brasília/DF. 2009.

COELLI, T. J.; RAO, D. S. P.; O’DONNELL, C. J.; \& BATTESE, G. E. An introduction to efficiency and productivity analysis. Springer Science \& Business Media, 2005.

DELGADO, V. M. S.; MACHADO, A. F. Eficiência das escolas públicas estaduais de Minas Gerais. 2007. Pesquisa e Planejamento Econômico, v. 37, n. 3, 2007. 
DINIZ, J. A.; CORRAR, L. J. Alocação de recursos públicos na educação fundamental: uma relação entre os gastos e desempenhos dos alunos da rede pública municipal. In: $\mathbf{1 1}^{\mathbf{o}}$ Congresso USP de Controladoria e Contabilidade. São Paulo/SP. 2011.

FARIA, F. P.; JANNUZZI, P. M.; SILVA, S. J. da. Eficiência dos gastos municipais em saúde e educação: uma investigação através da análise envoltória no estado do Rio de Janeiro. Revista de administração pública, v. 42, n. 1, p. 155-177, 2008.

FERNANDES, R. Índice de Desenvolvimento da Educação Básica (IDEB): metas intermediárias para a sua trajetória no Brasil, estados, municípios e escolas. Instituto Nacional de Estudos e Pesquisas Educacionais Anísio Teixeira, Brasília, 2007.

FONSECA, P. C.; FERREIRA, M. A. M. Investigação dos níveis de eficiência na utilização de recursos no setor de saúde: uma análise das microrregiões de Minas Gerais. Saúde e Sociedade, v. 18, n. 2, p. 199-213, 2009.

HAAS, D. J. Productivity efficiency of English football teams: A data envelopment analysis approach. Managerial and Decision Economics, v. 24, p. 403-410, 2003.

HAIR JUNIOR, J. F.; ANDERSON, R. E.; TATHAM, R. L.; \& BLACK, W. C. Análise multivariada de dados. 5. ed. Porto Alegre. Bookman, 2005.

LINS, M. P. E.; MEZA, L. A. Análise envoltória de dados e perspectivas de integração no ambiente do apoio à decisão. COPPE/UFRJ, 2000.

MARINHO, A. Avaliação da eficiência técnica nos serviços de saúde nos municípios do Estado do Rio de Janeiro. Revista brasileira de economia, v. 57, n. 3, p. 515-534, 2003.

MARTINS, G. de A.; THEÓPHILO, C. R. Metodologia da investigação científica para ciências sociais aplicadas. São Paulo: Atlas, 2007.

MELlO, J. C. C. B. S.; MEZA, L. A.; GOMES, E. G.; NETO, L. B. Curso de análise de envoltória de dados. Simpósio Brasileiro de Pesquisa Operacional, v. 37, 2005.

MINISTÉRIO da Fazenda - Tesouro Nacional. Finanças Brasil (FINBRA). Disponível em: <www.tesouro.fazenda.gov.br/estados-municipios/>. Acesso em: 14 jul. 2015.

PARO, V. H. Administração escolar: introdução crítica. São Paulo: Cortez, 2003. 
PORTELA, E. N. A Política de descentralização de recursos públicos para o ensino fundamental e seus reflexos na gestão da qualidade do ensino público municipal. 2006. Dissertação (Mestrado em Educação) - Universidade Católica de Brasília, Brasília, 2006.

REZENDE, A. J.; SLOMSKI, V.; CORRAR, L. J. A gestão pública municipal e a eficiência dos gastos públicos: uma investigação empírica entre as políticas públicas e o índice de desenvolvimento. Revista Universo Contábil, v. 1, n, 1, p. 24-40, 2005.

RIANI, F. Economia do setor público: uma abordagem introdutória. Atlas, 2002.

SAVIAN, M. P. G.; BEZERRA, F. M. Análise de eficiência dos gastos públicos com educação no ensino fundamental no estado do Paraná. Economia \& Região, v. 1, n. 1, p. 2647, 2013.

SCHMELKES, S. Buscando uma melhor qualidade para nossas escolas. Ministério da Educação e do Desporto, Secretaria de Educação Fundamental, 1994.

SILVA, A. A. P. Eficiência na Alocação de Recursos Públicos e Qualidade de Vida nos Municípios Mineiros. 2009. Dissertação (Mestrado em Administração) - Universidade Federal de Viçosa (UFV), Viçosa, 2009.

SILVA, A. A. P.; FERREIRA, M. A. M.; BRAGA, M. J.; ABRANTES, L. A. Eficiência na alocação de recursos públicos destinados à educação, saúde e habitação em municípios mineiros. Contabilidade, Gestão e Governança, v. 15, n. 1, 2012.

SILVA, A. C. Eficiência e equidade no ensino público fundamental nos municípios da Região Metropolitana de Salvador, Oeste baiano e Médio São Francisco: uma avaliação a partir de uma função de bem-estar social. Economia Baiana, 2008.

SILVA, C. M. D. da. Eficiência na alocação de recursos públicos na educação básica em Minas Gerais. 2013. Dissertação (Mestrado em Administração) - Universidade Federal de Lavras (UFLA), Lavras, 2013.

SILVA, M. C.; SOUZA, F. J. V.; ARAÚJO, A. O. Análise da eficiência dos gastos públicos com educação nas capitais brasileiras. ConTexto, v. 13, n. 24, p. 7-21, 2013.

SOUZA JÚNIOR, C. V. N. de; GASPARINI, C. E. Análise da eqüidade e da eficiência dos estados no contexto do federalismo fiscal brasileiro. Estudos Econômicos (São Paulo), v. 36, n. 4, p. 803-832, 2006. 
WILBERT, M. D.; D’ABREU, E. C. C. F. Eficiência dos gastos públicos na educação: análise dos municípios do estado de alagoas. Advances in Scientific and Applied Accounting, v. 6, n. 3, p. 348-372, 2013.

ZOGHBI, A. C. P.; MATOS, E. H. C. D.; ROCHA, F. F.; ARVATE, P. R. Mensurando o desempenho e a eficiência dos gastos estaduais em educação fundamental e média. Estudos Econômicos (São Paulo), v. 39, n. 4, p. 785-809, 2009. 